\title{
Determination of interplanetary coronal mass ejection geometry and orientation from ground-based observations of galactic cosmic rays
}

\author{
T. Kuwabara, ${ }^{1}$ J. W. Bieber, ${ }^{1}$ P. Evenson, ${ }^{1}$ K. Munakata, ${ }^{2}$ S. Yasue, ${ }^{2}$ C. Kato, ${ }^{2}$ \\ A. Fushishita, ${ }^{2}$ M. Tokumaru, ${ }^{3}$ M. L. Duldig, ${ }^{4}$ J. E. Humble, ${ }^{5}$ M. R. Silva, ${ }^{6}$ A. Dal Lago, ${ }^{6}$ \\ and N. J. Schuch ${ }^{7}$ \\ Received 2 September 2008; revised 10 March 2009; accepted 18 March 2009; published 27 May 2009.
}

[1] We have developed a method for determining interplanetary coronal mass ejection (ICME) geometry from galactic cosmic ray data recorded by the ground-based muon detector network. The cosmic ray density depression inside the ICME, which is associated with a Forbush decrease, is represented by an expanding cylinder that is based on a theoretical model of the cosmic ray particle diffusion. ICME geometry and orientation are deduced from observed time variations of cosmic ray density and density gradient and are compared with those deduced from a magnetic flux rope model. From March 2001 to May 2005, 11 ICME events that produced Forbush decreases $>2 \%$ were observed, and clear variations of the density gradient due to ICME passage were observed in 8 of 11 events. In five of the eight events, signatures of magnetic flux rope structure (large, smooth rotation of magnetic field) were also seen, and the ICME geometry and orientation deduced from the two methods were very similar in three events. This suggests that the cosmic ray-based method can be used as a complementary method for deducing ICME geometry especially for events where a large Forbush decrease is observed.

Citation: Kuwabara, T., et al. (2009), Determination of interplanetary coronal mass ejection geometry and orientation from groundbased observations of galactic cosmic rays, J. Geophys. Res., 114, A05109, doi:10.1029/2008JA013717.

\section{Introduction}

[2] Owing to the large detector mass required to detect high-energy cosmic rays, ground-based instruments remain the state-of-the-art method for studying these elusive particles. Muon detectors record secondary cosmic rays created by interactions of $>1 \mathrm{GeV}$ primary cosmic rays with Earth's atmosphere. These cosmic rays are the dominant source of ionization in Earth's atmosphere, especially in the upper troposphere. In addition, at energies up to $\sim 100 \mathrm{GeV}$, primary galactic cosmic rays experience significant variation in response to passing solar wind disturbances such as interplanetary coronal mass ejections (ICMEs).

[3] ICMEs and their accompanying shocks propagate through interplanetary space and may reach Earth. Some ICMEs, called magnetic clouds, have a rope-like magnetic structure [Burlaga et al., 1981; Klein and Burlaga, 1982],

\footnotetext{
${ }^{1}$ Bartol Research Institute and Department of Physics and Astronomy, University of Delaware, Newark, Delaware, USA.

${ }^{2}$ Physics Department, Shinshu University, Matsumoto, Japan. Japan.

${ }^{3}$ Solar-Terrestrial Environment Laboratory, Nagoya University, Nagoya,

${ }^{4}$ Australian Antarctic Division, Kingston, Tasmania, Australia.

${ }^{5}$ School of Mathematics and Physics, University of Tasmania, Hobart, Tasmania, Australia.

${ }^{6}$ National Institute for Space Research, São José dos Campos, Brazil.

${ }^{7}$ Southern Regional Space Research Center, CRS, INPE, MCT, Santa Maria, Brazil.
}

Copyright 2009 by the American Geophysical Union. 0148-0227/09/2008JA013717\$09.00 and such structures can be a factor in producing geomagnetic storms [Gosling et al., 1991; Zhang et al., 2007]. The ICME geometry and orientation at $1 \mathrm{AU}$ is of interest for understanding the interaction of the structure with Earth's magnetosphere, and has been determined using several methods. For example, fitting magnetic field observations to a model magnetic flux rope is perhaps the most commonly employed method for determining the magnetic field inside the ICME and the ICME geometry [Burlaga, 1988; Lepping et al., 1990; Farrugia et al., 1993; Leitner et al., 2007; Démoulin et al., 2008].

[4] Recently, a study has demonstrated that modeling the high-energy cosmic ray density inside the ICME can also be used to determine ICME magnetic field geometry and orientation [Kuwabara et al., 2004]. Behind the shock (if present) and inside the ICME, there is a cosmic ray densitydepleted region associated with a Forbush decrease [Cane, 2000; Hofer and Flueckiger, 2000], and that sometimes results in precursory signatures observable upstream of the shock [Munakata et al., 2005]. Within and around this depleted region, there is a " $\mathrm{B} \times \operatorname{grad}(\mathrm{n})$ " drift flow originating with the particle gyromotion and the density (n) gradient perpendicular to the magnetic field (B). This density gradient depends on the structure of the depleted region, and is calculated from the direction of drift flow and the interplanetary magnetic field (IMF) vector [Bieber and Evenson, 1998]. The method works by comparing the time variation of the density gradient with that expected on the basis of a theoretical model of the cosmic ray density 
depleted region [Munakata et al., 2006]. In this way we can determine the ICME geometry and orientation from the model calculation.

\section{Observation of the Cosmic Ray Density and Density Gradient}

[5] The cosmic ray density and the drift flow driven by a density gradient are measured by the prototype network of the ground based muon detector network. Three multidirectional muon detectors at Nagoya (Japan), Hobart (Australia), and São Martinho da Serra (Brazil) that operated from March 2001 to May 2005 are used in this work. (Readers can refer to Kuwabara et al. [2006] for detailed information on our detectors.) The energy response of our detectors varies with the inclination of each directional channel, thus the median rigidity of primary cosmic rays recorded for the different directions ranges from about fifty to one hundred GV. Pressure corrected hourly muon intensities recorded by our network are fitted to the function representing a firstorder anisotropy,

$$
\begin{aligned}
I_{i, j}^{f i t}\left(t_{i}\right)=I c_{0 i, j}^{0} & +\xi_{x}^{G E O}\left(c_{1 i, j}^{1} \cos \omega t_{i}-s_{1 i, j}^{1} \sin \omega t_{i}\right) \\
& +\xi_{y}^{G E O}\left(s_{1 i, j}^{1} \cos \omega t_{i}+c_{1 i, j}^{1} \sin \omega t_{i}\right) \\
& +\xi_{z}^{G E O} c_{1 i, j}^{0},
\end{aligned}
$$

where $I_{i, j}^{\text {fit }}$ is the intensity measured by the $j$ th directional channel in the $i$ th muon detector. This yields for each hour the best fit density, I, of primary cosmic rays (i.e., the omnidirectional component of intensity) as well as the three components of the streaming vector, or first-order anisotropy, in the geographic (GEO) coordinate system $\xi_{x}^{G E O}$, $\xi_{y}^{G E O}, \xi_{z}^{G E O}$. In equation $(1), t_{i}$ is the local time in hours at the $i$ th station, $\omega$ is $2 \pi / 24 \mathrm{~h}$, and $c_{0}^{0}$, etc., are so-called "coupling coefficients" [Fujimoto et al., 1984], which relate the observed muon intensity variation to the primary cosmic ray intensity variation in free space. We then obtain the anisotropy vector in the GSE coordinate system $\xi_{x}, \xi_{y}, \xi_{z}$ by a coordinate transformation. The rigidity dependence of the intensity variation can be measured by our detectors and is considered in the coupling coefficients. The derived value of $I$ is the value at $60 \mathrm{GV}$, which corresponds to the median rigidity of the vertical channel of the Nagoya detector. In contrast, the anisotropy vector $\xi_{x}, \xi_{y}, \xi_{z}$ is assumed to be rigidity-independent, which is consistent with the average diurnal anisotropy over a wide range of rigidity covered by the surface and underground muon detectors [Munakata et al., 1997].

[6] Following Bieber and Evenson [1998], we calculate the fractional density gradient $\boldsymbol{g}_{\perp}(t)$ perpendicular to the IMF from the derived anisotropy, as

$$
\mathbf{g}_{\perp}(t)=R_{L} \frac{\nabla_{\perp} N}{N}=-\mathbf{b}(t) \times \boldsymbol{\xi}^{w}(t)
$$

where $R_{L}$ is the particle Larmor radius (for a particle with $90^{\circ}$ pitch angle), and $\mathbf{b}(t)$ is a unit vector in the direction of the IMF. In this equation, the anisotropy in the solar wind frame $\boldsymbol{\xi}^{\boldsymbol{w}}(t)$ is derived from $\boldsymbol{\xi}(t)$ by subtracting the streaming due to the Compton-Getting effect of solar wind convection and earth orbital motion.

\section{Expanding Cylinder Model for Cosmic Rays}

[7] For the cosmic ray density gradient, we used the expanding-convecting cylinder model of Munakata et al. [2006], which is a refinement of the static convecting cylinder model of Kuwabara et al. [2004]. Note that an axisymmetric straight cylinder is considered here as a local approximation of the ICME.

[8] A model of the fractional density distribution and density gradient inside the ICME is derived from a transport equation,

$$
\frac{\partial f}{\partial t}=\frac{\kappa_{\perp}}{r} \frac{\partial}{\partial r}\left(r \frac{\partial f}{\partial r}\right)-V_{e x} \frac{\partial f}{\partial r}+\frac{1}{3 r} \frac{\partial}{\partial r}\left(r V_{e x}\right) \frac{\partial f}{\partial \ln p},
$$

where $f(r, p, t)$ is the omnidirectional phase space density at momentum $p$, a radial distance $r$ from the cylinder axis, and time $t$, and where $\kappa_{\perp}$ is the perpendicular diffusion coefficient and $V_{e x}$ is the radial expansion velocity of the cylinder (here taken to be independent of time).

[9] According to this model, the density depression $I(x)$ as a function of $x$, the distance from the cylinder axis normalized to the cylinder radius, is

$$
I(x)=a_{0}\left\{1+\frac{\Gamma}{4} x^{2}+\frac{\Gamma^{2}}{64} x^{4}+\cdots\right\},
$$

where $a_{0}$ is the density depression on the cylinder axis.

[10] The quantity $\Gamma$ is a dimensionless parameter related to $\kappa_{0}$ (see below) and the cosmic ray power spectrum index $\gamma$ as

$$
\Gamma=\frac{2(2+\gamma)}{3 \kappa_{0}}
$$

with spectral index here taken to be $\gamma=2.7$. The dimensionless parameter $\kappa_{0}$ is defined by the perpendicular diffusion coefficient $\kappa_{\perp}$, the cylinder radius $R$, and expansion speed $V_{e x}$ as,

$$
\kappa_{0}=\frac{\kappa_{\perp}}{R V_{e x}}
$$

Here we further assume that $\kappa_{\perp}$ is proportional to $R$, therefore $\kappa_{0}$ is time-independent.

[11] The cosmic ray density expected at Earth is derived from the model density distribution by assuming a vector $\mathbf{P}_{E}(t)$ that points from Earth to the Closest Axial Point (CAP) on the cylinder axis at time $t$. With this vector $\mathbf{P}_{E}(t)$ and cylinder radius $R(t)$, normalized radial distance at the location of Earth $x(t)$ becomes

$$
x(t)=\frac{\left|\mathbf{P}_{E}(t)\right|}{R(t)} .
$$

Next, the expected density $I^{\exp }(t)$ observed at Earth as a function of time $t$ is deduced from the spatial density distribution as

$$
I^{e x p}(t)=a_{0}\left\{1+\frac{\Gamma}{4} x(t)^{2}+\frac{\Gamma^{2}}{64} x(t)^{4}+\cdots\right\}
$$


and also the expected density gradient vector $\boldsymbol{g}_{\perp}^{\exp }(t)$ is deduced from $I^{\exp }(t)$ as

$$
\begin{aligned}
\mathbf{g}_{\perp}^{e x p}(t) & =-\frac{R_{L}}{R(t)} \frac{1}{I^{\exp }} \frac{d I^{\exp }}{d x} \mathbf{e}_{\perp}(t) \\
& =-\frac{R_{L}}{R(t)} \frac{a_{0}}{I^{\exp }}\left\{\frac{\Gamma}{2} x(t)+\cdots\right\} \mathbf{e}_{\perp}(t),
\end{aligned}
$$

where $\mathbf{e}_{\perp}(t)$ is the unit vector parallel to $\mathbf{P}_{E}(t)$.

[12] The vector $\mathbf{P}_{E}(t)$ is calculated from the velocity and orientation of the cylinder [Kuwabara et al., 2004]. We assume that the cylinder moves at the average solar wind speed inside the ICME, $\mathbf{V}_{s w}$, and that the axial direction is given by a unit vector $\mathbf{e}_{a x}$ that shows cylinder orientation. (Since either of two oppositely pointing vectors can define the cylinder axis, we adopt the convention that $\mathbf{e}_{a x}$ always points into the northern hemisphere.) Then, the vector $\mathbf{P}_{E}(t)$ is given by

$$
\mathbf{P}_{E}(t)=\left\{\mathbf{V}_{s w}-\left(\mathbf{e}_{a x} \cdot \mathbf{V}_{s w}\right) \mathbf{e}_{a x}\right\}\left(t-t_{c}\right)+\mathbf{P}_{c},
$$

where $t_{c}$ is the time when the cylinder is closest to Earth, and $\mathbf{P}_{c}$ defines the location of the CAP at the time of closest approach $t_{c}$, i.e., $\mathbf{P}_{c}=\mathbf{P}_{E}\left(t_{c}\right)$, and is derived by

$$
\mathbf{P}_{c}=d \frac{\mathbf{V}_{s w} \times \mathbf{e}_{a x}}{\left|\mathbf{V}_{s w} \times \mathbf{e}_{a x}\right|},
$$

where $d$ is the impact parameter, i.e, the distance between Earth and CAP at the time of closest approach $t_{c}$. As used here, the impact parameter $d$ is a signed quantity, with a negative value indicating that the CAP passed Earth on the dawnside, and a positive value indicating that it passed on the duskside. For a more detailed derivation, see Kuwabara et al. [2004]. Note that the quantity in braces in equation (10) corresponds to the apparent velocity $\mathbf{V}_{a p p}$ of Kuwabara et al. [2004]. Radius of the cylinder $R(t)$ is described by the expansion velocity $V_{e x}$ as

$$
\begin{gathered}
R(t)=R_{\text {in }}+V_{\text {ex }} \cdot\left(t-t_{\text {in }}\right) \\
V_{\text {ex }}=\frac{R_{\text {out }}-R_{\text {in }}}{t_{\text {out }}-t_{\text {in }}},
\end{gathered}
$$

where $R_{\text {in }}$ and $R_{\text {out }}$ are the radius of the cylinder at the times when Earth enters $\left(t_{i n}\right)$ and leaves $\left(t_{\text {out }}\right)$ the cylinder. These expressions follow from equation (10) because the absolute value of the vector $\mathbf{P}_{E}$ coincides with the cylinder radius when Earth touches its boundary.

[13] In this analysis, we use solar wind speed $\mathbf{V}_{s w}$ that is observed aboard the ACE satellite and averaged over the analyzed time period. We also use times that Earth encounters the cylinder boundaries, $t_{i n}$ and $t_{\text {out }}$, corresponding to the period of rapid variation of the density gradient. We also require that the minimum of the Forbush decrease should be included. The axis direction $\mathbf{e}_{a x}$ is replaced by a parameter defined by the GSE latitude $\theta$, and longitude $\phi$ of the axis direction. Munakata et al. [2006] took the axis direction from the value derived by the magnetic flux rope analysis, but in this analysis we set $\theta$ and $\phi$ as free parameters for the purpose of comparing with the one determined by the magnetic flux rope analysis. Then, cosmic ray density and density gradient at Earth are functions of seven parameters, $a_{0}, \kappa_{0}, R_{L}, d, \theta, \phi$, and $t_{c}$.

\section{ICME Geometry and Orientation}

[14] We chose eight ICME events to analyze and to determine their geometry. During a nearly 4 year period from March 2001 to May 2005 that our prototype network was in operation, we first selected 11 events that produced Forbush decreases with magnitude $>2 \%$. We discarded three events that did not display clear variations of the density gradient large enough to apply our method, leaving eight events for further analysis.

\subsection{The 29 October 2003 ICME}

[15] The 29 October 2003 ICME, part of the "Halloween, 2003" interval of enhanced solar activity, produced the largest Forbush decrease in our analysis period. Observations and results for this event are shown in Figure 1. Figure 1 (right) displays magnetic field and solar wind observations recorded by the ACE satellite. After the ICME-driven shock shown by the vertical solid line, the solar wind speed exceeds $1850 \mathrm{~km} / \mathrm{s}$ and IMF magnitude reaches $68 \mathrm{nT}$. Signatures of a magnetic flux rope (strong magnetic field and smooth rotation) are seen during the interval delimited by the vertical dotted lines. As additional signatures of ICME, though not shown in Figure 1, bidirectional electrons are also identified in this event, while the clear decrease of proton temperature frequently found in ICMEs was not present, and proton densities were uncertain [Skoug et al., 2004].

[16] Figure 1 (left) shows muon detector network observations of the cosmic ray density $I$, anisotropy vector $\boldsymbol{\xi}$, and density gradient $\boldsymbol{g}_{\perp}$. The vertical solid line shows the time of storm sudden commencement (SSC) associated with the shock arrival at $0611 \mathrm{UT}$, and we can see a $>10 \%$ Forbush decrease and the onset of strong anisotropy after the shock arrival. Latitude and longitude of the hourly mean IMF are plotted over the anisotropy as triangle marks, and the density gradient is determined from them. Rotation of the density gradient vector, especially rapid variation of the $g_{\perp x}$ component from negative to positive, produced by ICME passage, are observed during the period (1100 UT, 29 October to 0100 UT, 30 October) shown by vertical dotted lines.

[17] Nonlinear least squares fitting was applied to the observed density and three components of the density gradient vector. The residual $S$ of the best fit calculation is defined as

$$
S=\sqrt{\frac{1}{4 N} \sum_{i=1}^{N}\left\{\left|I^{o b s}\left(t_{i}\right)-I^{\exp }\left(t_{i}\right)\right|^{2}+\left|\mathbf{g}_{\perp}^{\text {obs }}\left(t_{i}\right)-\mathbf{g}_{\perp}^{\exp }\left(t_{i}\right)\right|^{2}\right\}}
$$

where $N$ is the number of data points between the vertical dotted lines, the superscript "exp" refers to the model expectations given in equations (8) and (9), and the superscript "obs" refers to the observed quantities shown in Figure 1. The red lines in Figure 1 show the model 

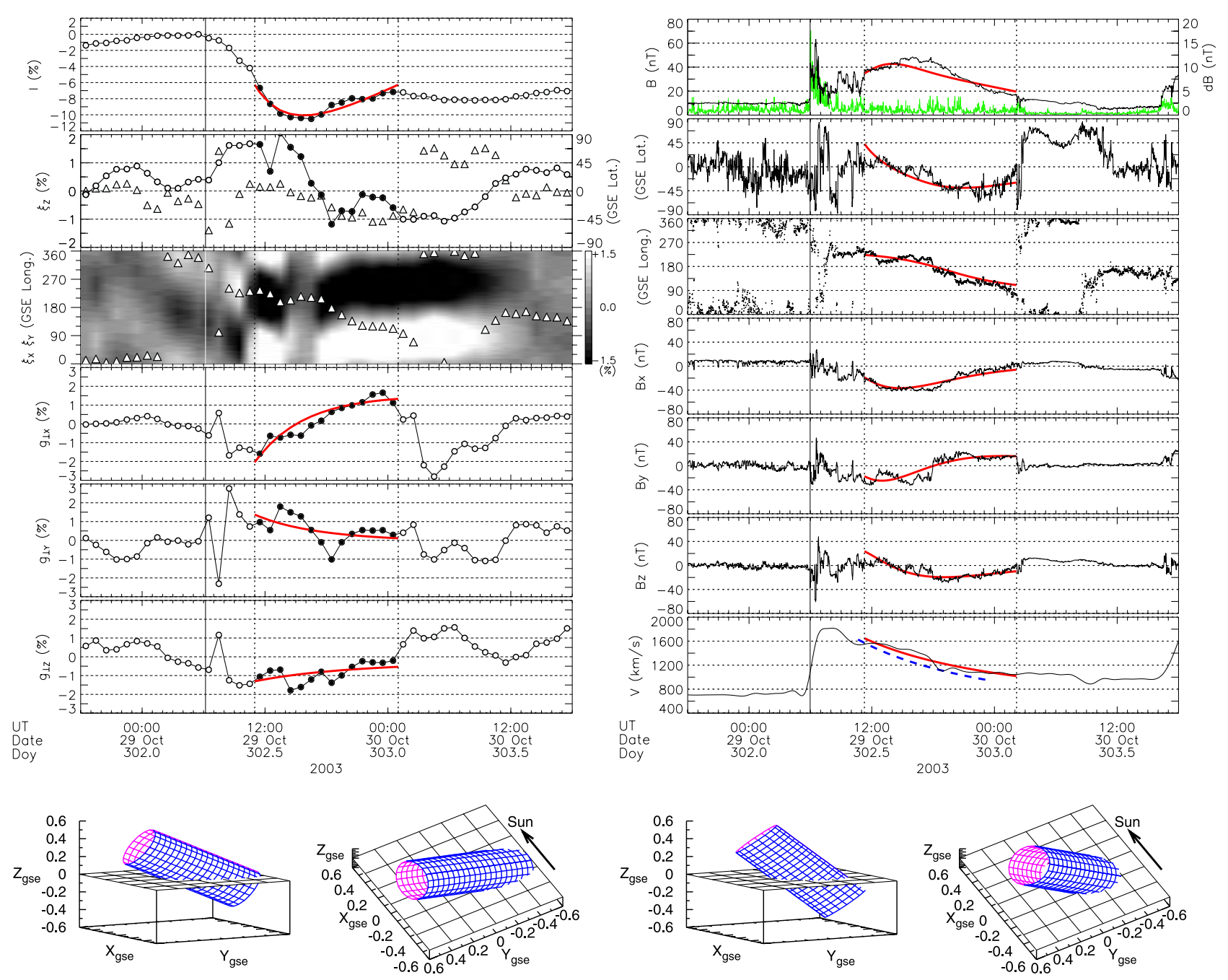

Figure 1. Observation and modeling of ICME geometry on 29 October 2003 (left) from cosmic ray density gradients determined from prototype muon detector network and (right) from magnetic flux rope model based upon ACE IMF measurements. Figure 1 (left) shows hourly value of the cosmic ray density, north-south anisotropy, the component anisotropy in the ecliptic plane in a gray scale format, and the three components of the density gradient in GSE coordinates. Latitude and longitude of the hourly mean IMF are plotted over the anisotropy as triangle marks in the second and third panels, respectively. In Figure 1 shows $64 \mathrm{~s}$ values of IMF magnitude, latitude, longitude, three Cartesian components of IMF, and the solar wind speed. The vertical solid line shows the arrival time of the shock, and dotted lines show ICME/flux rope boundaries. Timings that correspond to them at Earth and cosmic ray observation (SSC onset time, and boundaries of cosmic ray cylinder expected from observed density and density gradient) are also indicated in Figure 1 (left). In Figure 1 (bottom), the geometry in GSE coordinates deduced from both methods is compared.

predictions, and they do a reasonable job of producing the large-scale variation of the cosmic ray density and density gradient. Seven best fit parameters characterize the ICME model: $a_{0}, \kappa_{0}, R_{L}, d, \theta, \phi$, and $t_{c}$. Parameters related to cylinder inclination are $\theta=35^{\circ}$ and $\phi=78^{\circ}$. The cylinder radius at the time $t_{c}=302.63$ day of year (DOY) (1504 UT, 29 October) when the cylinder is closest to Earth is determined to be $R\left(t_{c}\right)=0.215 \mathrm{AU}$. The ICME orientation at $1 \mathrm{AU}$ expected from these parameters is illustrated in Figure 1 (bottom left).

[18] The ICME structure in this event is also determined from a magnetic flux rope calculation and is compared with the one from the cosmic ray model calculation. A constant $\alpha$ force-free cylindrical flux rope model including self-similar expansion [Farrugia et al., 1993; Marubashi, 1997] is used in this work. Boundaries of the flux rope are determined as 1117 UT, 29 October to 0210 UT, 30 October. The boundaries are generally determined following the criteria of Burlaga et al. [1981]: enhanced magnetic field strengths, smooth and large rotation of the magnetic field direction, low proton temperature, and low plasma beta. However, in some events including this event, no proton temperature and density data are available from the ACE satellite, so we primarily use magnetic field properties for the determination. We also look for reduction of the field fluctuation as an ICME signature [Cane and Richardson, 2003], $d B$, shown 
Table 1. Result of the Cosmic Ray Analysis for Eight Events ${ }^{\mathrm{a}}$

\begin{tabular}{|c|c|c|c|c|c|c|c|c|c|c|c|c|}
\hline Event Date & FD $(\%)$ & $a_{0}(\%)$ & $\kappa_{0}$ & $R_{L}(\mathrm{AU})$ & $\theta(\operatorname{deg})$ & $\phi(\mathrm{deg})$ & $t_{c}(\mathrm{UT})$ & $d(\mathrm{AU})$ & $S$ & $V_{e x}(\mathrm{AU} / \mathrm{d})$ & $R\left(t_{c}\right)(\mathrm{AU})$ & $\kappa_{\perp}\left(t_{c}\right)\left(\mathrm{cm}^{2} / \mathrm{s}\right)$ \\
\hline 4 Apr 2001 & 2.0 & -2.4 & 97 & 0.051 & 7 & 56 & $0112,5 \mathrm{Apr}$ & -0.068 & 0.3377 & -0.009 & 0.109 & - \\
\hline 11 Apr 2001 & 2.5 & -2.5 & 126 & 0.078 & 66 & 12 & $0314,12 \mathrm{Apr}$ & -0.002 & 0.1536 & -0.022 & 0.060 & - \\
\hline 28 Apr 2001 & 3.0 & -2.7 & 174 & 0.109 & 26 & 283 & $1916,28 \mathrm{Apr}$ & 0.020 & 0.2225 & -0.017 & 0.097 & - \\
\hline 6 Nov 2001 & 3.0 & -3.0 & 104 & 0.042 & 38 & 273 & $1829,6 \mathrm{Nov}$ & 0.038 & 0.1897 & -0.040 & 0.074 & - \\
\hline 29 Oct 2003 & 11.0 & -11.4 & 14 & 0.042 & 35 & 78 & 1504,29 Oct & 0.113 & 0.4663 & 0.271 & 0.215 & $2.05 \times 10^{21}$ \\
\hline 26 Jul 2004 & 4.5 & -4.6 & 24 & 0.029 & 5 & 303 & $0736,27 \mathrm{Jul}$ & 0.033 & 0.4924 & 0.161 & 0.096 & $9.48 \times 10^{20}$ \\
\hline 9 Nov 2004 & 2.5 & -2.4 & 106 & 0.048 & 44 & 187 & 0642,10 Nov & 0.039 & 0.2427 & 0.133 & 0.065 & $2.36 \times 10^{21}$ \\
\hline 21 Jan 2005 & 2.0 & -1.8 & 78 & 0.042 & 6 & 335 & 0348, 22 Jan & 0.010 & 0.3167 & 0.085 & 0.053 & $9.21 \times 10^{20}$ \\
\hline
\end{tabular}

${ }^{\mathrm{a}}$ Event date which is the date of SSC onset, observed Forbush decrease magnitude (\%), seven best fit parameters (density depression on the cylinder axis $a_{0}$, dimensionless perpendicular diffusion coefficient $\kappa_{0}$, particle Larmor radius $R_{L}$, GSE latitude $\theta$, and longitude $\phi$ of the cylinder axis, the time of closest approach $t_{c}$ and impact parameter $d$ ), residual $S$ of the best fit calculation, and additional parameters (expansion velocity $V_{e x}$, cylinder radius $R\left(t_{c}\right)$ and perpendicular diffusion coefficient $\kappa_{\perp}\left(t_{c}\right)$ at the time $\left.t_{c}\right)$.

as a green line in Figure 1 (top right), i.e., boundaries are chosen at the times corresponding to a transition from an ambient turbulent field to a strong and smooth rotating field, and vice versa. Red lines in Figure 1 (right) show predictions of the magnetic flux rope model, and they likewise do well at reproducing the observations. The inclination and radius of the magnetic flux rope from this model are $\theta_{m f r}=$ $-46^{\circ}, \phi_{m f r}=236^{\circ}$ and $R_{m f r}\left(t_{c}\right)=0.222$ AU with negative helicity. Angle difference of orientations obtained with the two models is estimated as $\Delta=20^{\circ}$. Note that antiparallel axis direction of $\theta_{m f r}$ and $\phi_{m f r}\left(46^{\circ}\right.$ and $\left.56^{\circ}\right)$ is used to calculate this value of $\Delta$. These values are very consistent with those determined from the cosmic ray modeling in this event.

[19] The orientation of this ICME has been also determined from ACE observations by several authors with different methods or models. Wang et al. [2005] used statistical force-free model [Lepping et al., 1990] and determined orientation as $\theta_{m f r}=-12^{\circ}$ and $\phi_{m f r}=246^{\circ}$ with negative helicity, from nearly same analysis period, $1100 \mathrm{UT}$, 29 October to 0230 UT, 30 October, as us. Inclination from the ecliptic place is smaller than the value determined from our flux rope model, but the angle difference from the cosmic ray method, $\Delta=26^{\circ}$, is still close to the orientation from cosmic ray method. Nieves-Chinchilla et al. [2005] used expanding force-free model but chose shorter period, 1100 UT, 29 October to 2310 UT, 29 October. Determined orientation is $\theta_{m f r}=55^{\circ}, \phi_{m f r}=125^{\circ}$, with positive helicity, and $\Delta=37^{\circ}$. Mandrini et al. [2007] used minimum variance method, and also chose nearly the same analysis period, 1125 UT, 29 October to 0200 UT, 30 October, as us; however, determined orientation is $\theta_{m f r}=-56^{\circ}, \phi_{m f r}=197^{\circ}$ $\left(\Delta=46^{\circ}\right)$ and is rather parallel to the GSE X-Z plane. From the Grad-Shafranov reconstruction technique $[\mathrm{Hu}$ et al., 2005; Yurchyshyn et al., 2005], similar orientation is determined to minimum variance method, with negative helicity at the period $1117 \mathrm{UT}, 29$ October to $0350 \mathrm{UT}, 30$ October. Hence, the cloud axis orientations derived from magnetic field data show considerable variation, presumably in part because of the different cloud boundaries assumed.

[20] Additional parameters that characterize the ICME are also determined from the cosmic ray analysis. The average particle Larmor radius inside the ICME (i.e., for those cosmic ray primaries contributing to the muon detector count rate) is determined from our model to be $R_{L}=$ $0.042 \mathrm{AU}$. The expansion velocity of the cylinder is $V_{e x}=$ $0.271 \mathrm{AU} / \mathrm{d}$, and the expected solar wind velocity from the cosmic ray analysis (computed by vectorically adding the observed average solar wind velocity to the derived expansion velocity) is shown by the blue dashed line in Figure 1 (right, bottom plot). The perpendicular diffusion coefficient at the time $t_{c}$ is determined to be $\kappa_{\perp}\left(t_{c}\right)=2.05 \times 10^{21} \mathrm{~cm}^{2} / \mathrm{s}$. From the measured in situ magnetic field intensity of $35.7 \mathrm{nT}$ averaged over the analyzed period, the Larmor radius is expected to be $0.037 \mathrm{AU}$ for a $60 \mathrm{GV}$ particle, which shows that the value inferred from our model is reasonable. The associated X-ray solar flare started at 0951 UT, 28 October (DOY 301.41), and the ICME passed Earth 1.22 days later, with an inferred radius $R\left(t_{c}\right)$ $=0.215 \mathrm{AU}$. This suggests the inferred expansion velocity $V_{e x}=0.271 \mathrm{AU} / \mathrm{d}$ is somewhat high (or varies with time). The perpendicular diffusion coefficient can also be estimated by other means. The ratio of parallel to perpendicular diffusion coefficients is theoretically calculated by Bieber et al. [2004] as $\kappa_{\perp} / \kappa_{\|} \sim 0.01$ for a $10 \mathrm{GV}$ proton and it becomes smaller at higher rigidity. The parallel diffusion coefficient has been estimated from longterm muon observations as $\kappa_{\|} \sim 3 \times 10^{23} \mathrm{~cm}^{2} / \mathrm{s}$ [Munakata et al., 2006]. Then, the perpendicular diffusion coefficient is estimated from these values as $\kappa_{\perp} \sim 3 \times$ $10^{21} \mathrm{~cm}^{2} / \mathrm{s}$ or lower, and is consistent with the value determined in our model. Note that the mean free path within the ICME is likely to be longer than average due to the exceptionally strong and smooth magnetic fields. Thus, the determined value might be regarded as an upper limit.

\subsection{Other Events}

[21] We investigated seven other ICME events in the same manner. Results of the cosmic ray analysis for all eight events including the 29 October 2003 ICME are summarized in Table 1. The observed size of Forbush decrease, best fit parameters $\left(a_{0}, \kappa_{0}, R_{L}, \theta, \phi, t_{c}\right.$ and $\left.d\right)$, residual $S$, and additional parameters $V_{e x}, R\left(t_{c}\right), \kappa_{\perp}\left(t_{c}\right)$ are determined and listed. We see that half of the events occurred during 2001 near solar maximum, while the two largest events including the 29 October 2003 ICME occurred during the descending phase of the solar cycle. By comparing with ACE magnetic field data during the period when cosmic ray analysis is performed, signatures of magnetic flux rope structure (smooth rotation of magnetic field) were noted in five events, but not in three events. ICME geometries determined from the two methods (cosmic ray and magnetic flux rope) are summarized and compared in Table 2. 
Table 2. Geometries and Orientations of ICME for Eight Events ${ }^{\mathrm{a}}$

\begin{tabular}{|c|c|c|c|c|c|c|c|c|c|}
\hline \multirow{3}{*}{ Event Date } & & \multirow{2}{*}{$\begin{array}{l}\text { Fit Begin to Fit End } \\
t_{\text {in }} \text { to } t_{\text {out }}\end{array}$} & \multirow{2}{*}{$\frac{\text { Closest Time }}{t_{c}}$} & \multicolumn{2}{|c|}{ Inclination } & \multicolumn{2}{|c|}{ Radius } & \multirow{2}{*}{$\frac{\text { CAP Location at Time } t_{c}}{\mathbf{P}_{c}(\mathrm{x}, \mathrm{y}, \mathrm{z} \text { in GSE })}$} & \multirow[b]{2}{*}{$\Delta^{\mathrm{c}}$} \\
\hline & & & & $\theta$ & $\phi$ & $R\left(t_{c}\right)$ & $|d|^{\mathrm{b}}$ & & \\
\hline & & $(\mathrm{UT})$ & (UT) & (deg) & $(\mathrm{deg})$ & $(\mathrm{AU})$ & $(\mathrm{AU})$ & $(\mathrm{AU})$ & $(\mathrm{deg})$ \\
\hline \multirow[t]{2}{*}{4 Apr 2001} & $\mathrm{CR}$ & 1900, 4 Apr to $0700,5 \mathrm{Apr}$ & 0112, $5 \mathrm{Apr}$ & 7 & 56 & 0.109 & 0.068 & $(-0.004,0.013,-0.067)$ & 54 \\
\hline & MFR & $1955,4 \mathrm{Apr}$ to $0658,5 \mathrm{Apr}$ & $0040,5 \mathrm{Apr}$ & 29 & 277 & 0.175 & 0.150 & $(0.000,-0.073,-0.131)$ & \\
\hline 11 Apr 2001 & $\mathrm{CR}$ & $2300,11 \mathrm{Apr}$ to $0700,12 \mathrm{Apr}$ & $0314,12 \mathrm{Apr}$ & 66 & 12 & 0.060 & 0.002 & $(0.000,0.002,0.000)$ & \\
\hline 28 Apr 2001 & $\mathrm{CR}$ & $00,28 \mathrm{Apr}$ to 01 & 1916, $28 \mathrm{Apr}$ & 26 & 283 & 0.097 & 0.021 & $(0.001,-0.009,-0.018)$ & \\
\hline 6 Nov 2001 & $\mathrm{CR}$ & 1400,6 Nov to $2200,6 \mathrm{Nov}$ & $1829,06 \mathrm{Nov}$ & 38 & 273 & 0.074 & 0.038 & $(0.000,-0.023,-0.030)$ & \\
\hline \multirow[t]{2}{*}{29 Oct 2003} & $\mathrm{CR}$ & 1100,29 Oct to 0100,30 Oct & 1504, 29 Oct & 35 & 78 & 0.215 & 0.113 & $(0.000,-0.066,0.091)$ & $20^{\mathrm{d}}$ \\
\hline & MFR & 1117,29 Oct to 0210,30 Oct & 1608,29 Oct & 46 & 56 & 0.222 & 0.103 & $(0.000,-0.080,0.064)$ & \\
\hline \multirow[t]{2}{*}{26 Jul 2004} & $\mathrm{CR}$ & $0400,27 \mathrm{Jul}$ to $1600,27 \mathrm{Jul}$ & $0736,27 \mathrm{Jul}$ & 5 & 303 & 0.096 & 0.033 & $(0.000,-0.004,-0.032)$ & $13^{\mathrm{d}}$ \\
\hline & MFR & $0141,27 \mathrm{Jul}$ to $1529,27 \mathrm{Jul}$ & 0742, 27 Jul & 16 & 296 & 0.136 & 0.0 & $(0.000,-0.004,-0.012)$ & \\
\hline \multirow[t]{2}{*}{09 Nov 2004} & $\mathrm{CR}$ & $0400,10 \mathrm{Nov}$ to $1600,10 \mathrm{Nov}$ & $0642,10 \mathrm{Nov}$ & 44 & 187 & 0.065 & 0.0 & $(0.001,-0.038,-0.004)$ & $10^{\mathrm{d}}$ \\
\hline & MFR & $0412,10 \mathrm{Nov}$ to $1634,10 \mathrm{Nov}$ & 0646, 10 Nov & 36 & 195 & 0.060 & 0.0 & $(0.000,-0.036,-0.013)$ & \\
\hline \multirow[t]{2}{*}{21 Jan 2005} & $\mathrm{CR}$ & 2300,21 Jan to $1800,22 \mathrm{Jan}$ & 0348, 22 Jan & 7 & 337 & 0.049 & 0.008 & $(0.000,-0.003,-0.009)$ & 75 \\
\hline & MFR & 2036,21 Jan to $1700,22 \mathrm{Jan}$ & 0220,22 Jan & 51 & 212 & 0.237 & 0.185 & $(0.000,-0.170,-0.074)$ & \\
\hline
\end{tabular}

${ }^{a}$ Event date, fitting interval, time of closest approach $t_{c}$, inclination (latitude $\theta$ and longitude $\phi$ in GSE), radius $R\left(t_{c}\right)$ of the cylinder, and impact parameter $|d|$, and CAP location at time of closest approach $P_{c}$, as deduced from cosmic ray-based method ("CR," eight events) and magnetic flux rope method ("MFR," five events). Angle difference $\Delta$ determined from the two methods for five events are also listed.

bImpact parameter.

${ }^{\mathrm{c}}$ Angle difference.

${ }^{\mathrm{d}}$ Events that yielded highly consistent ICME geometry and orientation (differences of $20^{\circ}$ or less between the axes derived from the two methods).
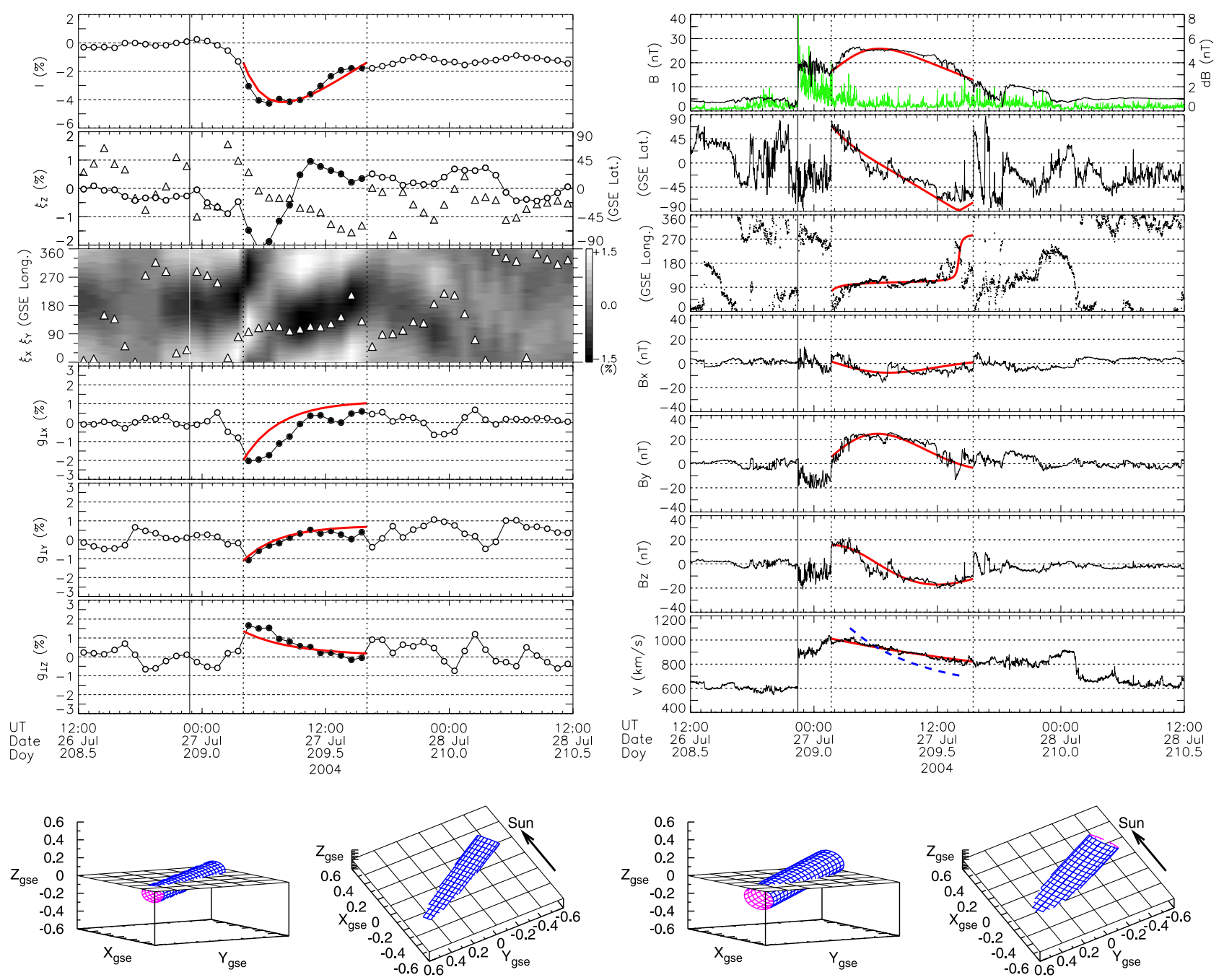

Figure 2. Observation and modeling of ICME geometry on 26-27 July 2004, displayed in the same manner as Figure 1. 

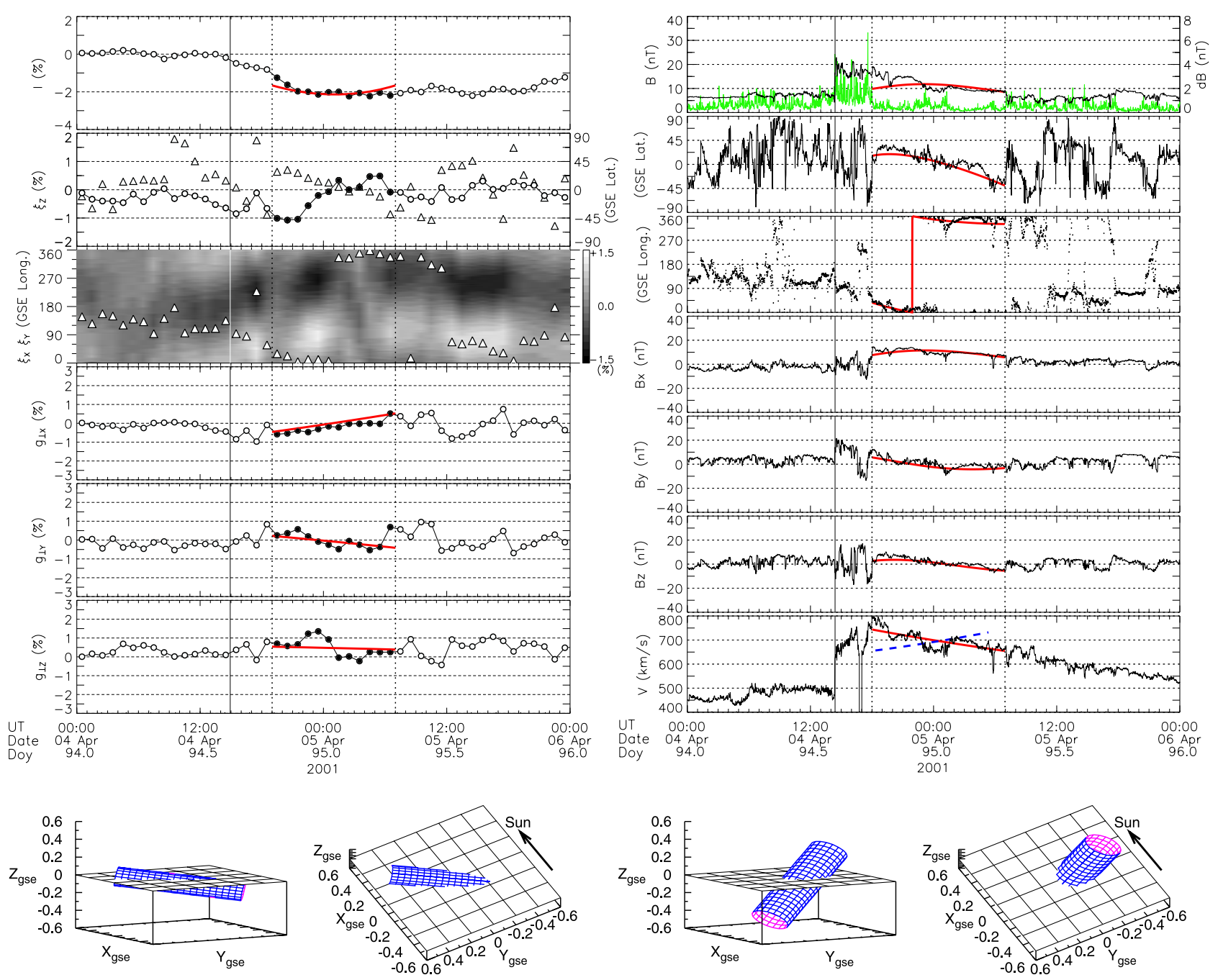

Figure 3. Observation and modeling of ICME geometry on 4-5 April 2001, displayed in the same manner as Figure 1.

[22] In the five events where both methods could be applied, ICME geometry and orientation deduced from the two methods agreed very well in three events (differences of $20^{\circ}$ or less between the axes derived from the two methods). The ICME of 26 July 2004 shown in Figure 2 is the second biggest and is one of the events with good agreement. The size of the Forbush decrease in this event was about half that of the 29 October 2003 event. However, large anisotropy and clear variations of density gradient produced by ICME passage are well observed in cosmic rays, and the derived inclination of the cosmic ray cylinder is similar to that derived from the magnetic flux rope analysis. The 4 April 2001 event shown in Figure 3 is one of the two events with poor agreement between the cosmic ray and MFR analysis. ICME signatures are seen in both analyses during the same period, however the derived geometries are dissimilar. Comparing with the events that did have good agreement, the rotation of the magnetic field was relatively small in this event. Thus, taking account of the field structure of the magnetic flux rope, it appears that the edge of the ICME passed the satellite and Earth, a circumstance that is also suggested by the ratio of the cylinder radius and the impact parameter determined from both analyses. It has been indicated that higher impact parameters tend to be derived when a satellite encounters an ICME which has significant curvature, and applying a cylinder model to those data is somewhat problematic [Marubashi, 2000]. In contrast, cosmic ray gradients determined from anisotropy observation result from the gyro motion of particles with fairly large gyroradii $\left(R_{L}=0.03-0.11 \mathrm{AU}\right)$ which sample the broad density gradient inside the ICME. We therefore suggest that the geometry and orientation deduced from cosmic ray analysis would be showing global structure of the ICME to a greater extent than the in situ observation of the local IMF.

[23] In the other three events, we could not do the magnetic flux rope analysis because the magnetic field variations did not display a clear flux rope structure during the period that cosmic ray analysis is performed. The 28 April 2001 event shown in Figure 4 is the one of those three events. Two ICMEs are evident in the observations of the magnetic field, and are indicated by a set of vertical dotted lines. Rotations of the cosmic ray density gradient vector are seen during the first ICME period, however rotations of 

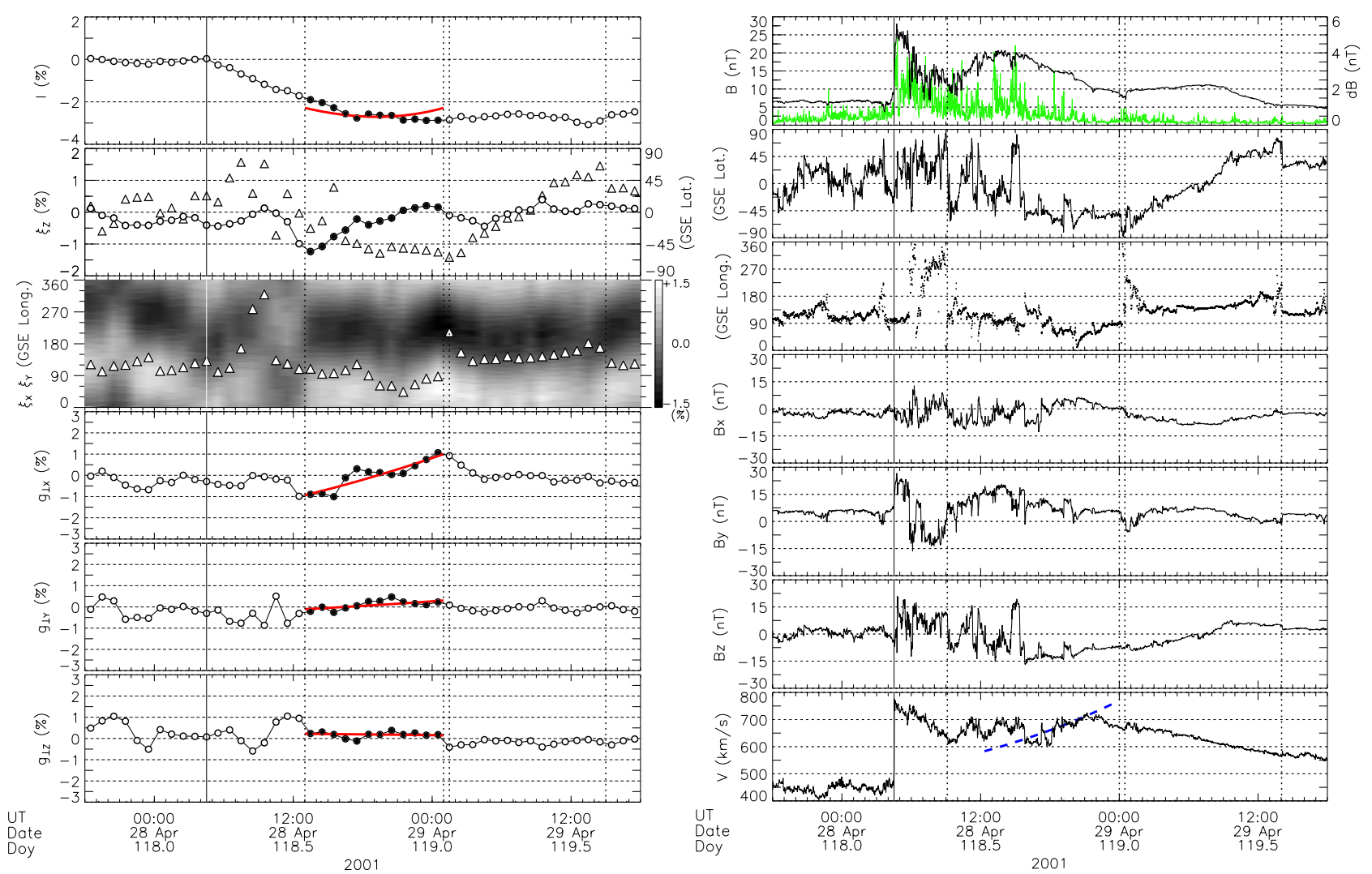

Figure 4. Observation and modeling of ICME geometry on 28 April 2001. (right) Two ICMEs are evident in the observations of the magnetic field and are indicated by a set of vertical dotted lines. (left) ICME signature is seen in cosmic ray data at first ICME; however the magnetic field variations did not display a clear flux rope structure during this period. The comparison of ICME geometries is not applied in this event.

magnetic field vector during this first interval do not have a flux rope character. Such unclear rotations of magnetic field were also seen in the events of 11 April 2001 and 6 November 2001. ICME signatures are still seen in cosmic rays in the subpart of the ICME, however the magnetic field variations did not display a clear flux rope structure during this period. This implies that the MFR analysis could not be performed for these events. In contrast, a gradient of cosmic ray density can often be generated even if the magnetic field disturbance is more complex than a magnetic flux rope. However, as shown by the second ICME in Figure 4, it can also happen that clear signatures of a flux rope are present with very little concurrent variation of the cosmic ray density or gradient. (Note however that the second ICME was weaker than the first one in terms of magnetic field strength.)

[24] Returning to the fit results presented in Tables 1 and 2 , the additional parameters $V_{e x}, R\left(t_{c}\right), \kappa_{\perp}\left(t_{c}\right)$ are also determined from our analysis. While the expansion velocity $V_{e x}$ was well determined in some events, nevertheless, small negative values are given in four events. As shown in Figures 1-3 (right, bottom plot), poor agreement between modeled (blue dashed line) and observed solar wind were seen in some events, possibly these values result not from the expansion of the cylinder but from an asymmetry of the density distribution inside the ICME, especially for the four events in 2001 where negative values may have resulted

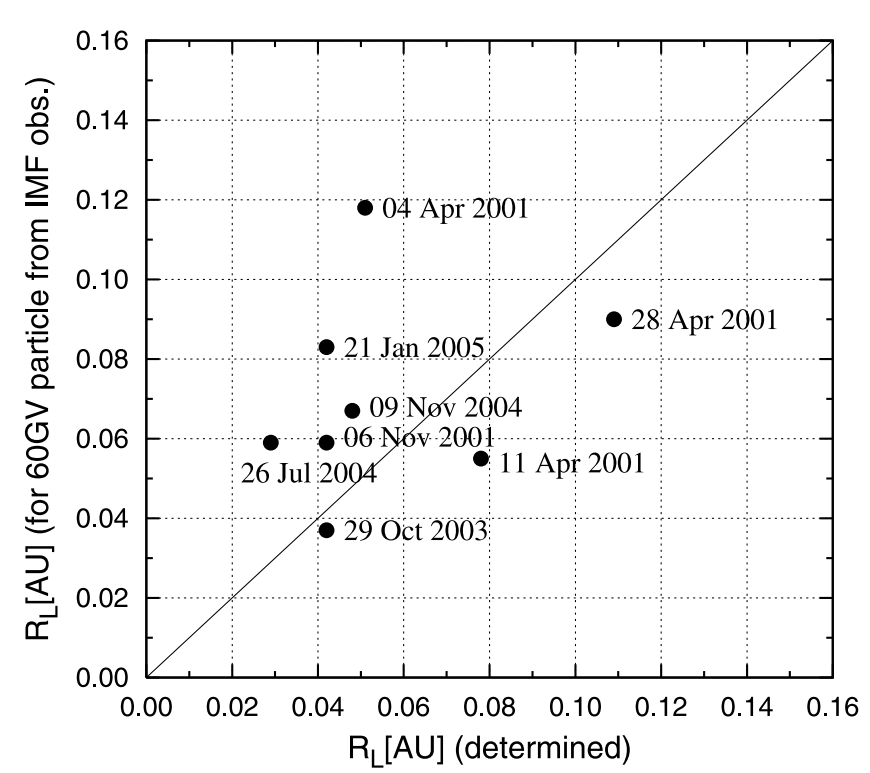

Figure 5. Particle Larmor radius determined from our analysis (abscissa) compared with expected value from in situ IMF measurements for a $60 \mathrm{GV}$ particle. 
because the first ICME is compressed or merged by the following one. On the other hand, reasonable values are determined for the cylinder radius $R\left(t_{c}\right)$ for all events. Cylinder radius $R\left(t_{c}\right)$ from the cosmic ray model ranges from 0.05 to $0.1 \mathrm{AU}$, except for $0.2 \mathrm{AU}$ for the large 29 October 2003 event. These are generally consistent with the value reported by Marubashi [2000]. The perpendicular diffusion coefficients $\kappa_{\perp}$ are derived for the four events that have positive $V_{e x}$, and they are less than or comparable to the value $2 \times 10^{21} \mathrm{~cm}^{2} / \mathrm{s}$ determined for 29 October 2003 .

[25] Figure 5 compares the particle Larmor radius $R_{L}$ determined from our analysis with the one expected for a $60 \mathrm{GV}$ particle given the IMF strength measured in situ and averaged over each analysis period. Most of them are not far from diagonal line where the two quantities are equal, but a majority have derived values somewhat smaller than expected based upon the IMF strength. We assume this effect results from the $P^{-\gamma}$ rigidity dependence of the Forbush decrease, where $\gamma$ ranges from about 0.4 to 1.2 [Cane, 2000], such that the effective energy of the particle deficit inside the ICME is lower than $60 \mathrm{GV}$ in these events. In fact, in the lower energy observations of the Spaceship Earth neutron monitor network [Bieber and Evenson, 1995] which observes $17 \mathrm{GV}$ median rigidity of primary cosmic rays, the decrease size was two times bigger than for muon observations in the 29 October 2003 event, and three times bigger in the 26 July 2004 event.

\section{Summary}

[26] A method for determining ICME geometry from galactic cosmic ray data has been developed based upon a model calculation of the cosmic ray density distribution. Nearly 4 years of data obtained from March 2001 to May 2005 were examined, and ICME geometry and orientation were deduced in eight events. In five of the eight events, signatures of magnetic flux rope structure were also seen, and results from the two methods were very similar in three events. In those three events, the derived inclination, radius, and impact parameters from the two methods were consistent with each other (differences of $20^{\circ}$ or less between the axes derived from the two methods), and the two biggest events on 29 October 2003 and on 26 July 2004 are among them. In the remaining two flux rope events, agreement between the methods was poor. These events displayed comparatively small magnetic field rotations, suggesting that Earth may have passed through the edge of the flux rope. In this circumstance, the flux rope analysis may be more sensitive to the local structure of the flux rope, whereas the cosmic ray analysis probes the global structure, owing to the large gyroradius of the cosmic ray particles which sample the broad density gradient inside the ICME.

[27] Finally, in the remaining three events, we could not do magnetic flux rope analysis because the magnetic field variations were not indicative of a flux rope structure, during the period that cosmic ray analysis was performed. Nonetheless the variations of cosmic ray anisotropy were large enough that meaningful gradients could be derived and modeled. The fit parameters obtained were reasonable and qualitatively similar to those obtained in the other events. This indicates that the cosmic ray-based method can be used as a new technique for deducing ICME geometry for events where a large Forbush decrease is observed but flux rope structure is not observed.

[28] The prototype network of multidirectional muon detectors has been upgraded to the Global Muon Detector Network (GMDN) in March 2006 by expanding the Brazilian detector in its detection area and adding a new detector in Kuwait University. The initial performance of the GMDN, which has been designed and constructed for the precise measurement of the anisotropy, can be found in our recent paper [Okazaki et al., 2008]. It is demonstrated in that paper that both the observation with the GMDN and a new analysis method enabled us to investigate the cosmic ray density gradient in three dimensions even in minor events due to corotational interaction regions during the period of minimum solar activity. The GMDN is now ready to observe major Forbush decreases expected to occur around the maximum period in solar cycle 24 .

[29] Acknowledgments. This work is supported by NASA grant NNX08AQ01G. The prototype network of the ground based muon detectors had been supported in part by a Grants-in-Aid for Scientific Research from the Ministry of Education, Culture, Sports, Science and Technology in Japan and by the joint research program of the Solar-Terrestrial Environment Laboratory, Nagoya University. We thank N. F. Ness for providing ACE magnetic field data via the ACE Science Center.

[30] Zuyin Pu thanks Ian Richardson and Stephen Kahler for their assistance in evaluating this paper.

\section{References}

Bieber, J. W., and P. Evenson (1995), Spaceship Earth-An optimized network of neutron monitors, Proc. Int. Cosmic Ray Conf., 24th(4), $1316-1319$.

Bieber, J. W., and P. Evenson (1998), CME geometry in relation to cosmic ray anisotropy, Geophys. Res. Lett., 25(15), 2955-2958.

Bieber, J. W., W. H. Matthaeus, A. Shalchi, and G. Qin (2004), Nonlinear guiding center theory of perpendicular diffusion: General properties and comparison with observation, Geophys. Res. Lett., 31, L10805, doi:10.1029/2004GL020007.

Burlaga, L., E. Sittler, F. Mariani, and R. Schwenn (1981), Magnetic Loop Behind an Interplanetary Shock: Voyager, Helios, and IMP 8 Observations, J. Geophys. Res., 86(A8), 6673-6684.

Burlaga, L. F. (1988), Magnetic clouds and force-free with constant alpha, J. Geophys. Res., 93(A7), 7217-7224.

Cane, H. V. (2000), Coronal mass ejections and Forbush decreases, Space Sci. Rev., 93(1/2), 55-77.

Cane, H. V., and I. G. Richardson (2003), Interplanetary coronal mass ejections in the near-Earth solar wind during 1996-2002, J. Geophys. Res., 108(A4), 1156, doi:10.1029/2002JA009817.

Démoulin, P., M. S. Nakwacki, S. Dasso, and C. H. Mandrini (2008), Expected in situ velocities from a hierarchical model for expanding interplanetary coronal mass ejections, Sol. Phys., 250(2), 347-374.

Farrugia, C. J., L. F. Burlaga, V. A. Osherovich, I. G. Richardson, M. P. Freeman, R. P. Lepping, and A. J. Lazarus (1993), A study of an expanding interplanetary magnetic cloud and its interaction with the Earth's magnetosphere: The interplanetary aspect, J. Geophys. Res., 98(A5), $7621-7632$.

Fujimoto, K., A. Inoue, K. Murakami, and K. Nagashima (1984), Coupling coefficients of cosmic ray daily variations for meson telescopes, Rep. 9, Cosmic Ray Res. Lab., Nagoya, Japan.

Gosling, J. T., D. J. McComas, J. L. Phillips, and S. J. Bame (1991), Geomagnetic activity associated with Earth passage of interplanetary shock disturbances and coronal mass ejections, J. Geophys. Res., 96, $7831-7839$.

Hofer, M. Y., and E. O. Flueckiger (2000), Cosmic ray spectral variations and anisotropy near Earth during the March 24, 1991, Forbush decrease, J. Geophys. Res., 105, 23,085-23,097.

Hu, Q., C. W. Smith, N. F. Ness, and R. M. Skoug (2005), On the magnetic topology of October/November 2003 events, J. Geophys. Res., 110, A09S03, doi:10.1029/2004JA010886.

Klein, L. W., and L. F. Burlaga (1982), Interplanetary Magnetic Clouds At 1 AU, J. Geophys. Res., 87(A2), 613-624.

Kuwabara, T., et al. (2004), Geometry of an interplanetary CME on October 29, 2003 deduced from cosmic rays, Geophys. Res. Lett., 31, L19803, doi:10.1029/2004GL020803. 
Kuwabara, T., et al. (2006), Real-time cosmic ray monitoring system for space weather, Space Weather, 4, S08001, doi:10.1029/2005SW000204.

Leitner, M., C. J. Farrugia, C. Möstl, K. W. Ogilvie, A. B. Galvin, R. Schwenn, and H. K. Biernat (2007), Consequences of the force-free model of magnetic clouds for their heliospheric evolution, J. Geophys. Res., 112, A06113, doi:10.1029/2006JA011940.

Lepping, R. P., J. A. Jones, and L. F. Burlaga (1990), Magnetic field structure of interplanetary magnetic clouds at 1 AU, J. Geophys. Res. 95(A8), 11,957-11,965.

Mandrini, C. H., M. S. Nakwacki, G. Attrill, L. van Driel-Gesztelyi, P. Démoulin, S. Dasso, and H. Elliott (2007), Are CME-Related Dimmings Always a Simple Signature of Interplanetary Magnetic Cloud Footpoints?, Sol. Phys., 244(1-2), 25-43, doi:10.1007/s11207-007-9020-8.

Marubashi, K. (1997), Interplanetary magnetic flux ropes and solar filaments, in Coronal Mass Ejections, Geophys. Monogr. Ser., vol. 99, edited by N. Crooker, J. Joselyn, and J. Feyman, pp. 147-156, AGU, Washington, D. C.

Marubashi, K. (2000), Physics of interplanetary magnetic flux ropes: Toward prediction of geomagnetic storms, Adv. Space Res., 26(1), 55-66.

Munakata, K., H. Miyasaka, D. L. Hall, S. Yasue, C. Kato, Z. Fujii, K. Fujimoto, and S. Sakakibara (1997), Long term variation of cosmic-ray diurnal anisotropy observed by a network of multi-directional muon telescopes in a wide range of rigidity, Proc. Int. Cosmic Ray Conf., 25th(2), 77.

Munakata, K., et al. (2005), A "loss cone" precursor of an approaching shock observed by a cosmic ray muon hodoscope on October 28, 2003 , Geophys. Res. Lett., 32, L03S04, doi:10.1029/2004GL021469.

Munakata, K., S. Yasue, C. Kato, J. Kota, M. Tokumaru, M. Kojima, A. A. Darwish, T. Kuwabara, and J. W. Bieber (2006), On the cross-field diffusion of galactic cosmic rays into an ICME, Adv. Geosci., 2, 115.

Nieves-Chinchilla, T., M. A. Hidalgo, and J. Sequeiros (2005), Magnetic clouds observed at 1 AU during the period 2000-2003, Sol. Phys. 232(1-2), 105-126, doi:10.1007/s11207-005-1593-5.

Okazaki, Y., et al. (2008), Drift effects and the cosmic ray density gradient in a solar rotation period: First observation with the global muon detector network (GMDN), Astrophys. J., 681, 693-707, doi:10.1086/588277.
Skoug, R. M., J. T. Gosling, J. T. Steinberg, D. J. McComas, C. W. Smith, N. F. Ness, Q. Hu, and L. F. Burlaga (2004), Extremely high speed solar wind: 29-30 October 2003, J. Geophys. Res., 109, A09102, doi:10.1029/ 2004JA010494.

Wang, Y., P. Ye, G. Zhou, S. Wang, S. Wang, Y. Yan, and J. Wang (2005), The interplanetary response to the great solar activities in late October 2003, Sol. Phys., 226(2), 337-357, doi:10.1007/s11207-005-6877-2.

Yurchyshyn, V., Q. Hu, and V. Abramenko (2005), Structure of magnetic fields in NOAA active regions 0486 and 0501 and in the associated interplanetary ejecta, Space Weather, 3, S08C02, doi:10.1029/ 2004SW000124.

Zhang, J., et al. (2007), Solar and interplanetary sources of major geomagnetic storms (Dst $\leq-100 \mathrm{nT}$ ) during 1996-2005, J. Geophys. Res., 112, A10102, doi:10.1029/2007JA012321.

J. W. Bieber, P. Evenson, and T. Kuwabara, Bartol Research Institute, University of Delaware, 217 Sharp Laboratory, Newark, DE 19716, USA (jwbieber@bartol.udel.edu; evenson@udel.edu; takao@bartol.udel.edu)

A. Dal Lago and M. R. Silva, National Institute for Space Research, Avenida dos Astronautas 1758, São José dos Campos, SP 12 227-010, Brazil. (dallago@dge.inpe.br; marlos@lacesm.ufsm.br)

M. L. Duldig, Australian Antarctic Division, Kingston, Tas 7050, Australia. (marc.duldig@aad.gov.au)

A. Fushishita, C. Kato, K. Munakata, and S. Yasue, Physics Department, Shinshu University, Matsumoto 390-8621, Japan. (s07t303@shinshu-u. ac.jp; ckato@shinshu-u.ac.jp; kmuna00@shinshu-u.ac.jp; shyasue@shinshuu.ac.jp)

J. E. Humble, School of Mathematics and Physics, University of Tasmania, Hobart, Tas 7001, Australia. (john.humble@utas.edu.au)

N. J. Schuch, Southern Regional Space Research Center, CRS, INPE, MCT, PB 5021, 97110-970, Santa Maria, RS, Brazil. (njschuch@aries. lacesm.ufsm.br)

M. Tokumaru, Solar-Terrestrial Environment Laboratory, Nagoya University, Nagoya, Aichi 464-8601, Japan. (tokumaru@stelab.nagoya-u.ac.jp) 